\title{
Innovación tecnológica y comunicativa para combatir la desinformación: 135 experiencias para un cambio de rumbo
}

\section{Technological and communicative innovation to fight misinformation: 135 experiences for a change of direction}

\author{
Jorge Vázquez-Herrero; Ángel Vizoso; Xosé López-García
}

Cómo citar este artículo:

Vázquez-Herrero, Jorge; Vizoso, Ángel; López-García, Xosé (2019). “Innovación tecnológica y comunicativa para combatir la desinformación: 135 experiencias para un cambio de rumbo". El profesional de la información, v. 28, n. 3, e280301.

https://doi.org/10.3145/epi.2019.may.01

Artículo recibido el 09-01-2019

Aceptación definitiva: 21-03-2019

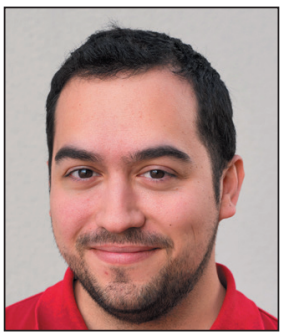

Jorge Vázquez-Herrero $\bowtie$ https://orcid.org/0000-0002-9081-3018

Universidade de Santiago de Compostela Facultade de Ciencias da Comunicación Campus Norte, Avda. de Castelao, s/n. 15782 Santiago de Compostela (A Coruña), España jorge.vazquez@usc.es

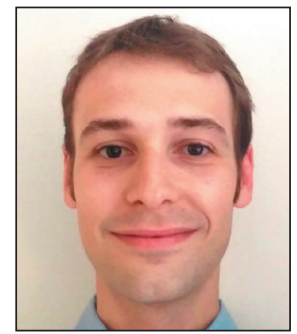

Ángel Vizoso https://orcid.org/0000-0001-7898-9267

Universidade de Santiago de Compostela Facultade de Ciencias da Comunicación Campus Norte, Avda. de Castelao, s/n. 15782 Santiago de Compostela (A Coruña), España angel.vizoso@usc.es

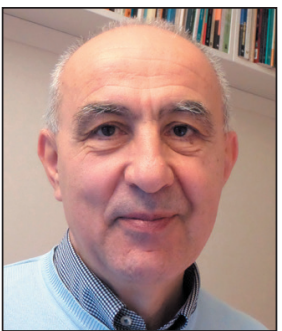

Xosé López-García

https://orcid.org/0000-0002-1873-8260

Universidade de Santiago de Compostela Facultade de Ciencias da Comunicación

Campus Norte, Avda. de Castelao, s/n. 15782 Santiago de Compostela (A Coruña), España xose.lopez.garcia@usc.es

\section{Resumen}

El impacto social de la desinformación en la sociedad red, alimentado por el efecto de las denominadas fake news, ha encendido numerosas luces de alarma en la segunda década del tercer milenio. La gran cantidad de mensajes que circula por las infraestructuras tecnológicas y las estrategias aplicadas para que alcancen el mayor impacto en los comportamientos sociales han incrementado la complejidad del escenario de la ubicuidad comunicativa. Esta situación ha sido interpretada desde el periodismo como una amenaza para la credibilidad y como un desafío para buscar antídotos que, mediante el cultivo del periodismo de calidad, ofrezcan respuestas a los ciudadanos interesados en la información veraz. En este trabajo se analizan 135 iniciativas de fact-checking de todo el mundo y se profundiza en la caracterización de seis casos seleccionados. Los resultados identifican modelos diferenciados en la organización, el contenido y las fórmulas de verificación para un cambio de rumbo.

\section{Financiación}

Este texto está elaborado en el marco del proyecto Cibermedios nativos digitales en España: formatos narrativos y estrategia móvil (RTI2018-093346-B-C33), del Ministerio de Ciencia, Innovación y Universidades". Este proyecto está cofinanciado por el fondo estructural Feder. Forma parte también de las actividades promovidas a través de la Red Internacional de Gestión de la Comunicación - Xescom (Referencia: ED341D R2016/019), apoyada por la Consellería de Cultura, Educación e Ordenación Universitaria de la Xunta de Galicia.

Los autores Ángel Vizoso y Jorge Vázquez-Herrero son beneficiarios del Programa de Formación del Profesorado Universitario financiado por el Ministerio de Ciencia, Innovación y Universidades (Gobierno de España). 


\title{
Palabras clave
}

Desinformación; Noticias falsas; Falsos; Chequeo; Verificación; Fact-checking; Posverdad; Ciberperiodismo; Periodismo digital; Comunicación; Perfiles profesionales; Fact-checkers.

\begin{abstract}
The social impact of misinformation in the network society, fueled by the effect of the so-called fake news, has awaken numerous warning lights in the second decade of the third millennium. The large number of messages that circulate through technological infrastructures and the strategies applied for them to achieve the greatest impact on social behavior have increased the complexity of the communicative ubiquity context. This situation has been interpreted by journalism as a threat to credibility and as a challenge to seek antidotes that offer answers to citizens interested in truthful information, through the cultivation of quality journalism. This article analyzes 135 fact-checking initiatives around the world and delves into the characterization of six selected cases. The results identify different models in the organization, the content and the verification formulae for a change of direction.
\end{abstract}

\section{Keywords}

Misinformation; Fake news; False information; Fact-checking; Verification; Post-truth; Digital journalism; Communication.

\section{Introducción}

Las técnicas de desinformación han estado muy presentes a lo largo de la historia de la Comunicación, especialmente durante los grandes conflictos bélicos y en las etapas de entreguerras (Bloch, 1999). Su impacto, que siempre ha sido muy relevante, nunca había alcanzado un lugar preferente en el debate público hasta la segunda década del tercer milenio, lo que hizo que el diccionario Oxford eligiese fake news como palabra del año en 2017.

La proliferación de fake news, en especial mediante piezas seudoperiodísticas, aumentó intensidad a las crisis cíclicas del periodismo, sumando gravedad al escenario de crisis económica y el proceso de adaptación de la industria al escenario digital construido alrededor de la galaxia internet (Castells, 2001). Los falsos han marcado la evolución del periodismo y han socavado su credibilidad con casos muy conocidos en alguno de los grandes diarios de referencia (Jones-Patterson; Urbanski, 2006). Esta técnica de comunicación social ha construido sus principios básicos o elementos esenciales sobre la verificación (Kovach; Rosenstiel, 2001), que sigue formando parte del núcleo de principios éticos que guían la profesión periodística en el s. XXI (McBride; Rosenstiel, 2013).

El periodismo ha vivido una fase de constantes cambios, más intensos desde la aparición de internet, la Web y el ciberperiodismo entendido como la especialidad del periodismo que emplea el ciberespacio para investigar, elaborar $y$, sobre todo, difundir mensajes informativos (Salaverría, 2005). La propia evolución del ciberespacio, a la sombra de la web social, ha abierto desde la segunda mitad de la primera década del siglo XXI renovadas vías para la participación de los ciudadanos en la construcción de los mensajes periodísticos (Paulussen et al., 2007), lo que introduce nuevas voces mediatizadas y abre desafíos en la producción y verificación de la información.

\section{Estado del arte}

La evolución tecnológica ha cambiado radicalmente el periodismo (Pavlik, 2000). El cambio permanente se ha instalado en los medios de comunicación, impulsado por la digitalización de los procesos informativos, lo que ha transformado las rutinas profesionales de los periodistas (Micó-Sanz; Pérez-Soler, 2015). Además, se han abierto nuevas vías para la producción (Hermida; Thurman, 2008) y el consumo de los contenidos (Casero-Ripollés, 2012). La mediamorfosis mediática (Fidler, 1997) ha desembocado en un ecosistema comunicativo líquido (Bauman, 2007). En él, la reinvención del periodismo avanza gracias a numerosas iniciativas que buscan vitaminas en el periodismo heredado y en la experimentación (Salaverría, 2015), en los nuevos soportes y mediante renovadas técnicas narrativas.

Del proceso de cambios vividos han resultado algunas de las principales transformaciones, que van desde la producción multimedia (Deuze, 2004; George-Palilonis, 2012) hasta el contenido generado por los usuarios y la participación de las audiencias activas (Singer, 2005; Carpentier, 2011; Holton; Lewis; Coddington, 2016), entre otros. Esta vía ha abierto nuevos desafíos para la verificación sin cambiar el rumbo de la participación. Los esfuerzos por encontrar caminos se basan, a juzgar por las experiencias de los últimos años en los principales cibermedios, en la combinación de la participación y los valores del periodismo (Hujanen, 2016), la articulación efectiva de la carga emocional de la información para intervenir en el nuevo escenario (Beckett; Deuze, 2016) y en la empatía entre periodista y usuario (Glück, 2016). Todo ello además de otras dimensiones y características que emergen en el presente con el convencimiento de que el futuro del periodismo es en red (Van-der-Haak; Parks; Castells, 2012). 
En medio de muchos debates e incertezas (Franklin, 2011) y con multitud de desafíos en un escenario caracterizado por un sistema híbrido de viejos y nuevos medios (Chadwick, 2013), han surgido propuestas para contribuir a un cambio de rumbo en el periodismo hacia la calidad y la credibilidad mediante refuerzos en el proceso de verificación.

Frente a las fake news ha aparecido el fact-checking, una práctica periodística basada tanto en la comprobación a posteriori de informaciones publicadas por los medios de comunicación, como en la verificación de las declaraciones o comentarios realizados por líderes políticos o personajes relevantes (Mantzarlis, 2018). Todo ello con el objetivo de evitar o mitigar los efectos de la proliferación de las noticias falsas (Gueham, 2017), cuyo tráfico ha experimentado un gran crecimiento en los últimos años debido a su fácil difusión a través de las redes sociales (Lopez-Borrull; Vives-Gràcia; Badell, 2018) y el anonimato que éstas posibilitan (Berinsky, 2015).

En 1995 nació Snopes.com, la primera iniciativa especializada íntegramente en fact-checking (Graves, 2016, p. 28). Desde ese momento, y especialmente a lo largo de la última década, han aparecido numerosos proyectos basados en la verificación de la información, tanto independientes como asociados a medios ya consolidados (Cherubini; Graves, 2016). En ellos la práctica profesional está basada en la idea de la investigación y verificación de los hechos desde su origen, acercándolos al público y aumentando su conocimiento (Elizabeth, 2014). Aunque la verificación de la información es una de las características básicas de la producción periodística, la calidad de este proceso está muchas veces en entredicho. Esto se debe tanto a la aceleración constante del trabajo informativo que ha traído consigo internet (Currie-Sivek; Bloyd-Peshkin, 2018), como a la facilidad para la difusión de informaciones falsas en las redes sociales en un momento en el que el consumo informativo se ha incrementado notablemente en esas plataformas (Zubiaga et al., 2016). Para frenar esta difusión, plataformas como Facebook y Twitter han llevado a cabo iniciativas dirigidas a identificar los contenidos falsos y los verificados (Wardle; Derakhshan, 2017). Del mismo modo, se han puesto en marcha herramientas cuyo objetivo es identificar cuentas automatizadas dirigidas a difundir desinformación (Roth, 2018).

En este contexto ha emergido la figura profesional del fact-checker, un periodista encargado de comprobar todos los datos de las informaciones publicadas y los discursos revisados en busca de posibles incoherencias o inexactitudes (Graves, 2016 , p. 77). Gracias al uso de técnicas tradicionales como la búsqueda en archivos documentales o el empleo de aplicaciones tecnológicas (Brandtzaeg et al., 2018) es posible contrastar y verificar los datos, imágenes u otros documentos que constituyen una información. Todo ello con el objetivo de certificarla o, en su caso, desmentirla aclarando dónde se encuentran las inexactitudes y aportando datos originales que construyan un relato verídico. Según Mantzarlis (2018), el proceso de verificación consta de tres fases:

1. Búsqueda de declaraciones verificables a través de la consulta de grabaciones parlamentarias, medios de comunicación y redes sociales.

2. Búsqueda de los hechos originales mediante la consulta de la mejor fuente disponible que los apoye.

3. Corrección del contenido mostrando las pruebas disponibles, para lo cual suele emplearse una escala de veracidad.

El fact-checking es una especialidad basada en la verificación o comprobación de datos con herramientas actuales -con la informática y las tecnologías como elementos básicos en el marco de actuación-. Es una respuesta ideada por el periodismo para combatir las noticias falsas y el incremento de su circulación a través de las redes sociales, con la finalidad de ofrecer a la ciudadanía un relato completamente ajustado a la realidad.

\section{Método}

Este artículo parte del objetivo de identificar el desarrollo de las iniciativas actuales de fact-checking en el mundo: caracterización organizacional, enfoque y fórmulas de verificación, con la aplicación de la triangulación metodológica (Gaitán-Moya; Piñuel-Raigada, 1998) para realizar un estudio exploratorio global y un estudio de caso, combinando la perspectiva cuantitativa y cualitativa (Bryman, 2006).

La investigación parte de la base de datos mundial de iniciativas de fact-checking del Duke Reporters' Lab ${ }^{1}$-un total de 227 (Duke Reporters' Lab, 2018)- y los medios adheridos a la Red Internacional de Verificación de la Información ${ }^{2}$ que coordina el Poynter Institute -con 54 (Poynter, 2018)-. Los datos fueron recogidos en noviembre de 2018 y, tras eliminar los duplicados, quedaron 236 iniciativas.

En primer lugar, se comprobó la última actividad registrada en cada uno de los sitios. Aquellos que no tuvieron actualización en los últimos 3 meses fueron descartados. Finalmente, la muestra quedó compuesta por 135 iniciativas $^{3}$. Dicha relación fue sometida a un análisis formal y de contenido a partir de una ficha que recoge: denominación, url, fecha de revisión, continente y país, última actividad, soporte asociado, medio o sección, naturaleza periodística o cívica, pertenencia, año de inicio, entidad financiadora, temática principal, ámbito geográfico que abarca, tipos de falsos que aborda, y fórmula de verificación que emplea. 
En una segunda fase se han seleccionado seis iniciativas para un estudio de caso centrado en las características singulares de los fact-checkers. Los criterios de selección tienen por objeto representar modelos diferentes, tanto por diversidad idiomática y geográfica como incluyendo iniciativas independientes y asociadas a medios de referencia. Tras la exploración realizada de los 135 sitios, los casos seleccionados son:

- $B B C$ reality check: pertenece a una televisión, medio de referencia, originario de Reino Unido;

- Chequeado: referente en español, especializado y online, pionero, pertenece a una fundación argentina;

- Fact-checker: pertenece a The Washington Post, referente de Estados Unidos;

- Les décodeurs: pertenece a Le monde, en Francia;

- Pagella politica: nació en Italia en 2012, es una asociación independiente de periodistas;

- Snopes: primer medio especializado, online.

El estudio de caso valora los aspectos contextuales de nacimiento de la iniciativa, organización, soporte y aspectos formales, centrándose en el método de verificación y la fórmula para comunicar el veredicto.

\section{Exploración mundial de iniciativas de fact-checking}

En el conjunto de iniciativas de verificación de la información alrededor del mundo hay un elevado número de sitios inactivos: el $29,07 \%$ según los datos del Duke Reporters' Lab (2018) y un 33,47\% según el criterio de esta investigación -actividad en los últimos 3 meses-e incluyendo los sitios vigilados por el Poynter Institute, después de descartar las 22 ediciones por Estado de Politifact que desde Duke contabilizan individualmente. Entre las 135 iniciativas activas, la moda $M=0$ sobre los días transcurridos desde la última actividad registrada señala que el caso más frecuente es que el medio se actualice diaria-

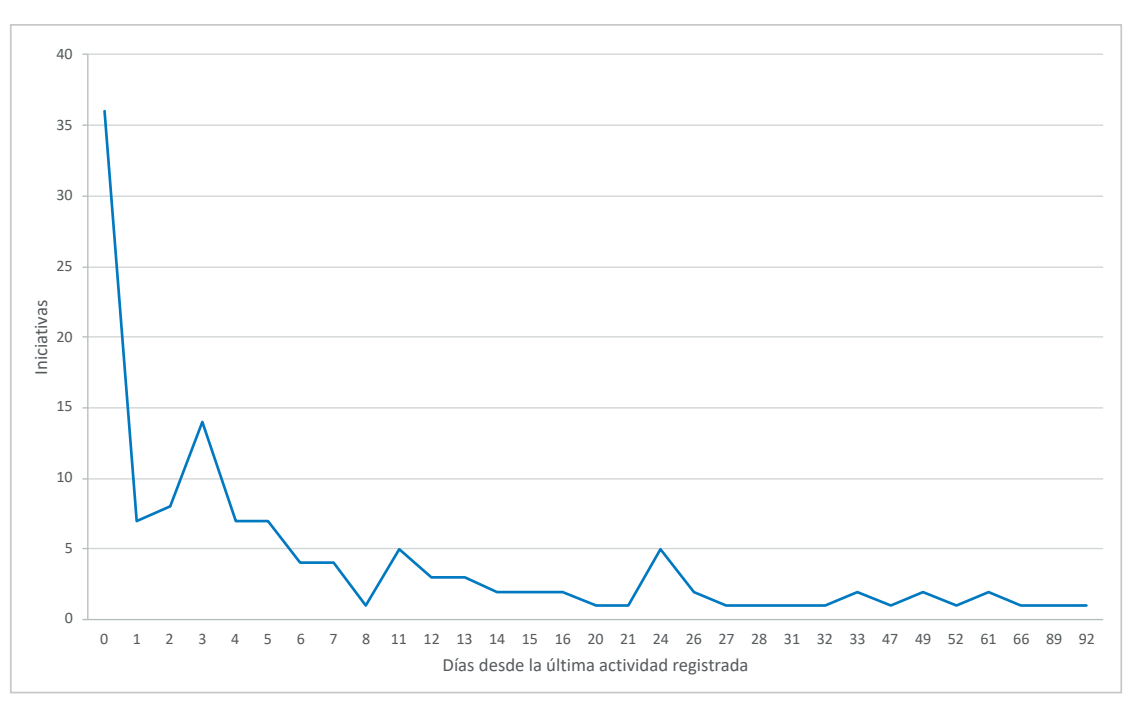

Figura 1. Última actividad registrada en los fact-checkers mente. El 64,44\% de los sitios ana-

lizados cuentan con publicaciones nuevas en la última semana. Sin embargo, en la muestra se localiza un 9,63\% que no se ha actualizado en el último mes y un 11,1\% que se ha actualizado entre 2 y 4 semanas atrás.

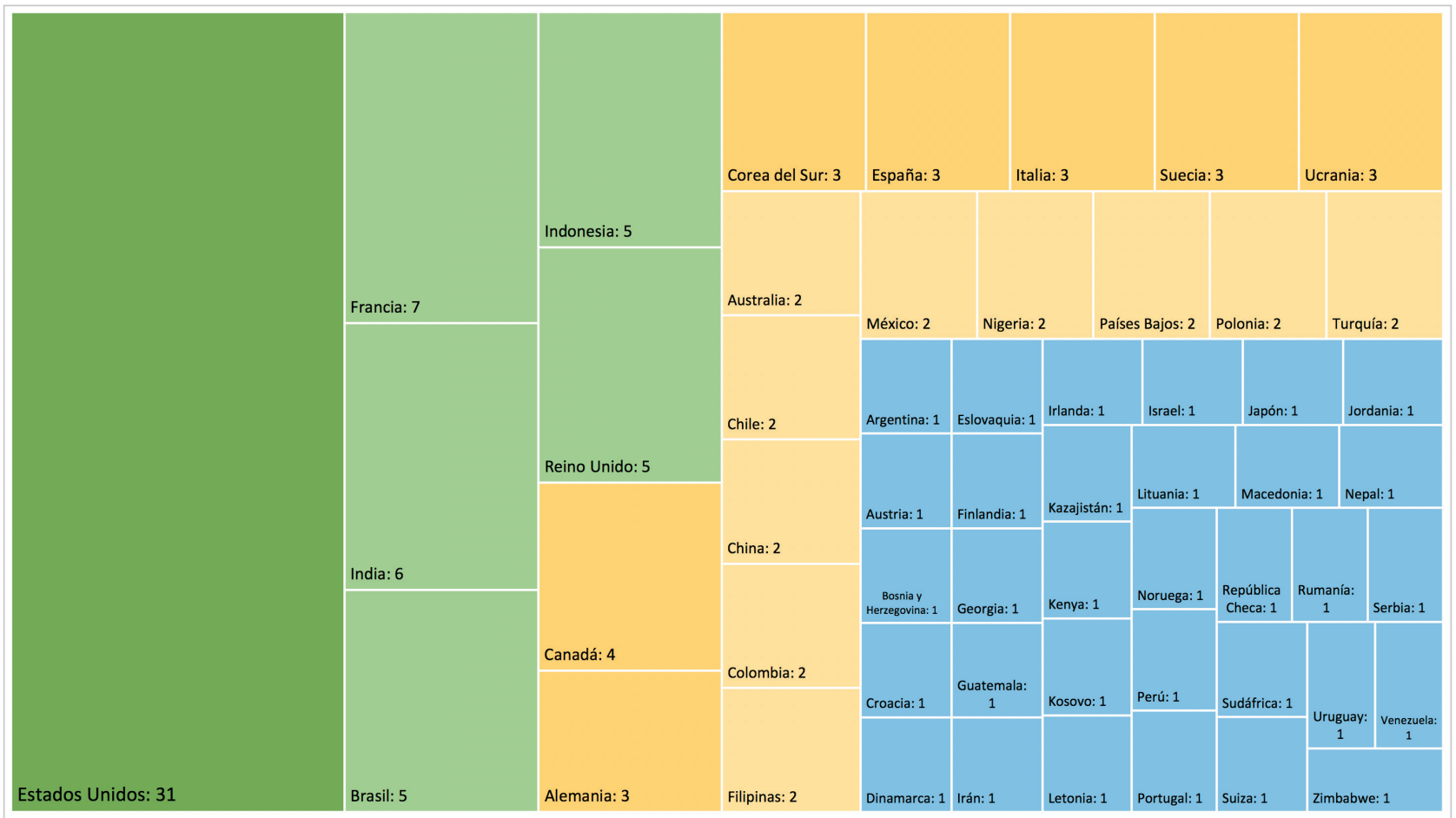

Figura 2. Número de iniciativas activas de fact-checking por países 
Atendiendo al origen geográfico, Europa -con 51 iniciativas- es el continente con mayor representación, aunque también se identifican casos en Norteamérica, Asia, Sudamérica, África y Oceanía. El país con mayor número de plataformas de verificación es Estados Unidos -31 en total-, junto a Francia, India, Brasil, Indonesia y Reino Unido, todos ellos con al menos 5 entidades de fact-checking.

Todas las plataformas de verificación cuentan con un sitio web, a excepción de El polígrafo de Chile. No obstante, algunas de ellas están asociadas a medios con matriz impresa, canal de televisión o emisora de radio. El 70,37\% (95 iniciativas) son nativas digitales, de ellas, 4 son agencias de noticias -la canadiense Science-Presse, Agência Lupa de Brasil, AGI de Italia y Associated Press-. Entre los medios tradicionales que toman parte en el fact-checking se encuentra la radiotelevisión pública de Letonia, National public radio, Radio France, la revista danesa Mandag morgen y televisiones como France 24, canales vinculados a la CBS, Channel 4 o laSexta.

Los sitios de verificación registrados se clasifican entre aquellos que son medios en sí mismos $(49,63 \%)$ y los que son secciones dentro de proyectos mayores $(50,37 \%)$. Repartidos de forma casi pareja, los medios especializados en fact-checking son pioneros y surgen de la actividad ciudadana. En 1995 nació Snopes y en 2002 Slovo i dilo y E-farsas; de los 67 medios, 22 se fundaron antes de 2014 -principalmente en Estados Unidos y, en el conjunto, predomina la lengua inglesa-. En cuanto a las secciones de verificación de la información, 16 nacieron antes de 2014, siendo las primeras en 2007 y con carácter periodístico: News3 reality check de WISC-TV, Les observateurs de France 24 y Fact-checker de The Washington Post. Por tanto, las plataformas pioneras fueron principalmente de naturaleza cívica e independientes de instituciones o medios. Sin embargo, de las 135 plataformas analizadas, el 69,63\% son periodísticas, debido a la alta proliferación posterior en los medios y entre periodistas.

Entre 2014 y 2017 surgió un gran número de iniciativas de fact-checking, especialmente en 2016, cuando se registran 26 nuevos sitios. Este boom se corresponde con la extensión del debate sobre la posverdad y las fake news con carácter global.

\section{El fact-checker se alía con la informática y la tecnología para dar una respuesta periodística a los falsos}

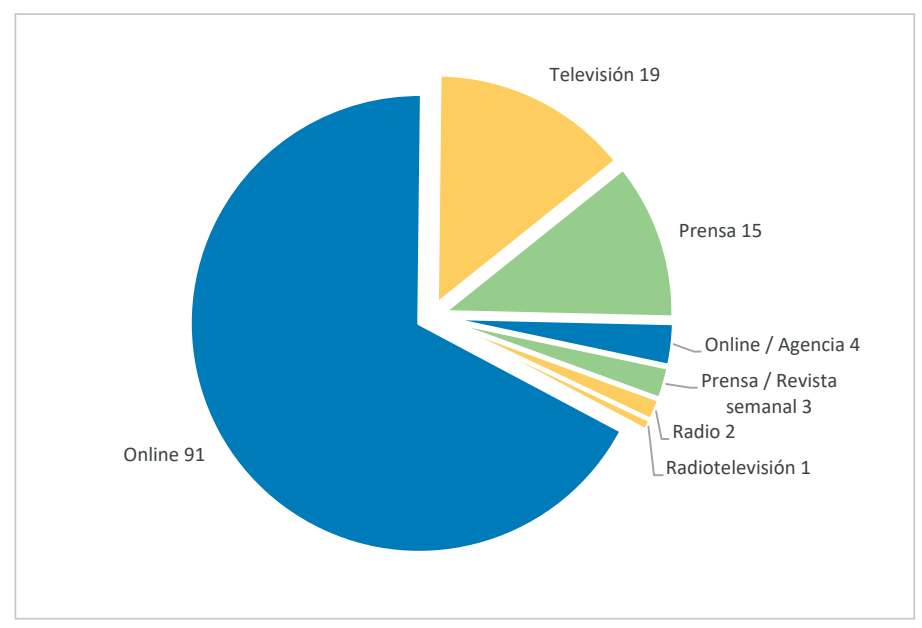

Figura 3. Tipos de soporte de las iniciativas de fact-checking activas

Estas iniciativas trabajan en su mayoría (60,74\%) sobre información de su país. No obstante, el 30,37\% son de ámbito internacional y un $8,89 \%$ lo hacen en su región -caso de aplicación a las plataformas de fact-checking centradas en alguno de los estados de Estados Unidos-. En cuanto al tema principal que abordan, el $62,22 \%$ son generalistas, aunque destaca el tercio de iniciativas centradas en cuestiones políticas -declaraciones de autoridades, debates, promesas electorales y análisis de los programas-. Más singulares resultan los 3 medios especializados en información científica -Climate feed-back, Science Vs, Détecteur de rumeurs- y Gossip cop sobre celebridades.

El tipo de noticias más comunes que verifican son promesas electorales, entrevistas y debates, declaraciones de políticos y personajes públicos o chequeo de datos aportados en medios o intervenciones. Sin embargo, también se registran

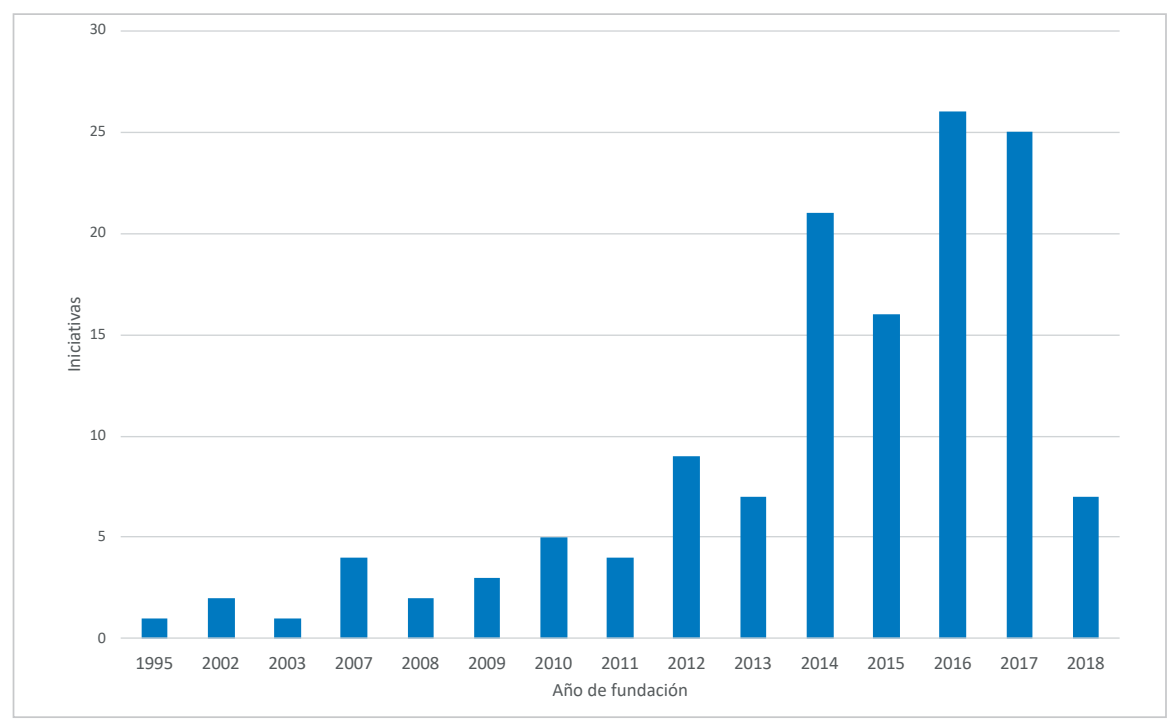

Figura 4. Evolución temporal de la emergencia de iniciativas de fact-checking 


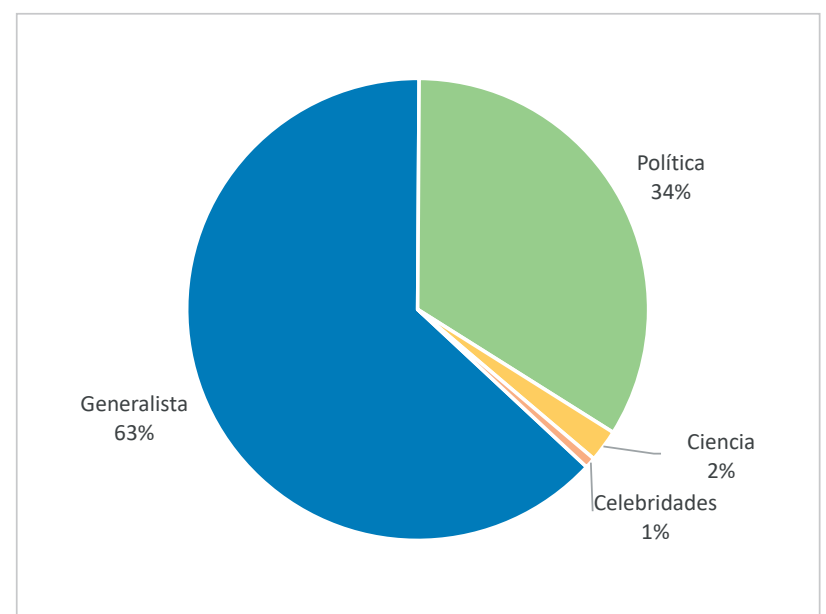

Figura 5. Temática principal de los fact-checkers

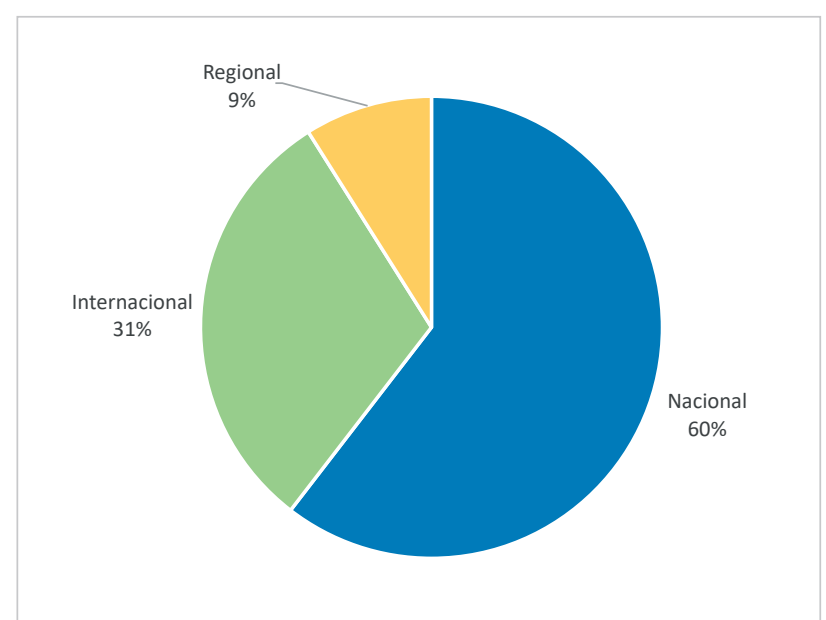

Figura 6. Ámbito de cobertura de los fact-checkers

verificaciones sobre contenido difundido en redes sociales y propuestas de los usuarios. En general, cada medio presenta diferente tipo de contenido; algunos se especializan en bulos, como Boatos en Brasil, Viralgranskaren en Suecia o Maldito bulo en España; y son numerosos los casos centrados en el discurso y el programa político: LuiPresident en Francia, Pagella politica en Italia o El sabueso en México.

Las 135 iniciativas analizadas difieren en el tipo de contenido que verifican, pero especialmente en la fórmula que emplean para comunicar su veredicto y evitar así la propagación de información falsa. El 61,48\% emplea algún recurso visual de escala, sello o símbolo. Este tipo de elementos facilitan la identificación e interpretación del veredicto emitido por la organización. Destaca el uso de termómetros, semáforos y velocímetros como metáforas que traducen el resultado de la verificación de forma visual y universal, así como las escalas que algunos medios en particular El máximo auge de iniciativas de fact-checking se situó entre 2014 y 2017 emplean: los pinochos de Fact Checker o el Truth-o-me-

ter y Trump-o-meter de Politifact. De esta forma, representan la cualidad de verdadero o falso con matices, a través de recursos reconocibles y comprensibles de manera ágil, en ocasiones con un sesgo humorístico.

La fórmula de verificación más común es la etiqueta cromática, que indica de forma concisa el veredicto en el propio titular, sobre la imagen principal o en el desarrollo del texto. El vídeo es el medio principal para argumentar la verificación en el $11,85 \%$ de los casos, generalmente vinculado a secciones en programas televisivos, donde el propio presentador desarrolla la argumentación con datos, documentos y gráficos. Por último, cabe destacar el widget Share the facts, contribución del Duke Reporters' Lab y la incubadora de tecnología Jigsaw de Alphabet: una ficha sintética que identifica los datos básicos y el veredicto, y permite ser compartida con facilidad en un formato adaptado al móvil. Este recurso ha sido implementado por diferentes sitios y en la indexación en Google recibe una posición preferente.

\section{Diferentes modelos para un mismo objetivo}

Las seis iniciativas estudiadas en profundidad en este artículo - BBC Reality check, Chequeado, Les décodeurs, Pagella politica, Snopes.com y Fact checker - constituyen un claro ejemplo de las diferentes estrategias empresariales y de producción existentes en el área de la verificación de la información. Encontramos organizaciones que funcionan fundamentalmente como medios independientes a pesar de que sus integrantes realizan cada vez más colaboraciones en otros medios - Chequeado, Pagella politica y Snopes.com-y otras integradas en medios de comunicación de referencia. Este sería el caso de BBC reality check - British Broadcasting Corporation-, Les décodeurs - Le monde- y Fact checker The Washington Post-, secciones encargadas de realizar trabajos de verificación para diferentes plataformas integradas en los medios. Por ejemplo, aunque la cabeza más visible de la $B B C$ es el medio televisivo, el espacio $B B C$ reality check elabora piezas exclusivas para la web, en las que se combina la información textual con contenidos multimedia como el vídeo, la fotografía y la infografía, aprovechando así todo el potencial de esta plataforma.

Algo semejante ocurre con las otras dos iniciativas pertenecientes a medios de comunicación. Los trabajos de Les décodeurs y de Fact checker están presentes en las páginas de los diarios a los que pertenecen. Sin embargo, es en la web donde han encontrado un acomodo mejor gracias a la posibilidad de combinar elementos de diversa naturaleza. Éstos dan lugar a piezas multimedia en las que la verificación de la información se sustenta sobre la aportación y la interpretación de las fuentes originales, bien de forma textual, bien mediante contenidos audiovisuales o conjuntos de datos.

La otra cara de la moneda es la de las tres iniciativas concebidas para internet. Chequeado, Pagella politica y Snopes nacieron en 2010, 2012 y 1994 respectivamente como cibermedios especializados en el fact-checking. Las tres son organizaciones independientes fundadas por ciudadanos sin bagaje periodístico cuya formación se centraba en áreas como 
la ciencia política, la economía, el derecho o las administraciones públicas, entre otras. De este modo:

- Snopes inició (de la mano de Barbara y David Mikkelson) el camino de los cibermedios especializados en la verificación, erigiéndose como la primera iniciativa especializada en fact-checking de la que se tiene constancia (Graves, 2016).

- Chequeado, plataforma argentina fundada en febrero de 2010 por Julio Aranovich, José Alberto Bekinschtein y Roberto Lugo, constituye la primera experiencia de fact-checking en español.

- Pagella política, surgida de la colaboración inicial de diez socios fundadores, fue hasta 2016 la única organización italiana especializada en la verificación de la información.

Dentro del plano organizativo encontramos tres modelos de financiación:

- BBC reality check, Les décodeurs y Fact checker pertenecen a medios o grupos mediáticos de los que proviene su financiación;

- las iniciativas independientes de Chequeado, PageIla politica y Snopes están financiadas por su propia actividad - proveedor de contenido a clientes, como Pagella politica-;

- y por la colaboración ciudadana a través de donaciones - fundamental en el caso de Snopes-

\section{La verificación: características y soportes empleados}

Las seis organizaciones elegidas ejemplifican los diferentes modos de abordar la verificación de la información, respecto a las características de los contenidos publicados y a los soportes empleados para su difusión.

\section{$B B C$ reality check}

https://www.bbc.com/news/topics/cp7r8vg/2rgt/ reality-check

Es una sección que presta apoyo a los contenidos publicados por el equipo de noticias del ente público británico. A partir de un titular a modo de pregunta, el equipo expone qué hay de cierto en las declaraciones o rumores más actuales. El texto es el elemento conductor e incluyen vídeos, fotografías, gráficos e hipervínculos para acceder a los contenidos originales que se referencian. Por tanto, esta iniciativa opta por una presentación de los contenidos similar a cualquier cibermedio informativo, estrategia que complementa con la difusión a través de @BBCRealityCheck en Twitter.

\section{Chequeado \\ https://chequeado.com}

Opta también por un enfoque similar al de cualquier cibermedio informativo, tanto en la disposición general de los contenidos como en la propia estructura formal de las piezas de verificación. Los "chequeos" se inician con un titular identificando autor y declaración, que será refutada, verificada o precisada a lo largo del texto, elemento que guía las verificaciones y que es complementado con imágenes o vídeos de los personajes públicos argentinos que realizaron las declaraciones, además de hipervínculos a fuentes oficiales. La difusión de los contenidos producidos se realiza también a través de Twitter y Facebook.

\section{Les décodeurs}

https://www.lemonde.fr/les-decodeurs

Es el nombre que Le monde da a su sección de verifi-

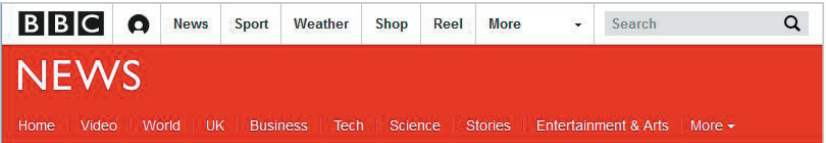

Reality Check
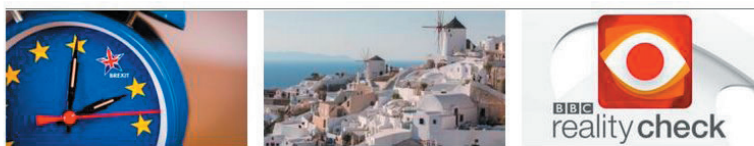

How the Brexit timetable could How cooling are white roofs? unfold

(i)

Q 3d

Q 2d

at do you want BBC Reality Check to investigate?

China's precious metals the US can't do without

(4) $4 \mathrm{~d}$

What is the minimum wage? 12 April 201

https://www.bbc.com/news/topics/cp7r8vgl2rgt/reality-check

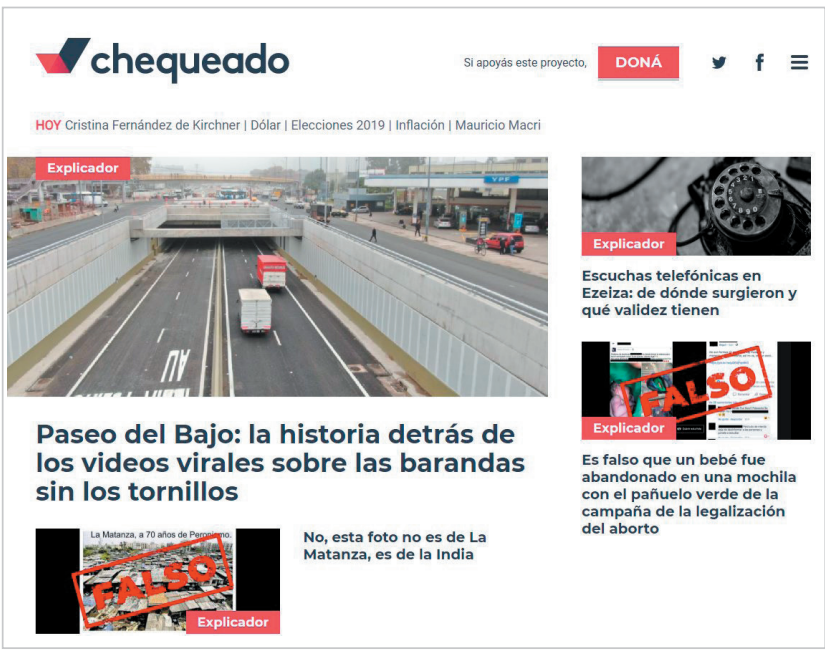

https://chequeado.com

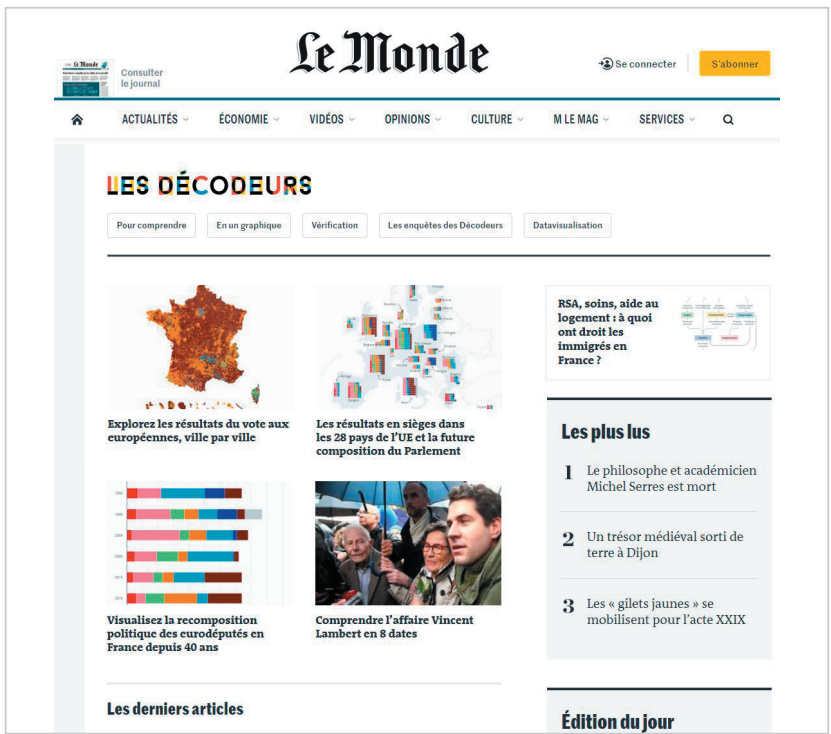

https://www.lemonde.fr/les-decodeurs 
cación y visualización de la información. En el sitio web del medio el usuario puede acceder a la aplicación Décodex y obtener el grado de fiabilidad de lo publicado en una url determinada. Además, la decena de periodistas del departamento verifica aquellos rumores que circulan por las redes en vrai faux, siguiendo la estructura clásica de noticia complementada con elementos entre los cuales destaca la infografía. Estos contenidos son difundidos también a través de Twitter, Facebook y Snapchat.

\section{Pagella politica}

https://pagellapolitica.it

Muestra una distribución muy semejante a Chequeado. Analizan las citas emitidas por los personajes públicos mediante textos enriquecidos con aportaciones como las fuentes originales y gráficos. Como particularidad, al final de cada pieza destaca la posibilidad de que los usuarios compartan la verificación consultada en las redes sociales. La iniciativa italiana está presente en Twitter, Facebook y, desde diciembre de 2018, en Instagram, además de ofrecer un servicio de suscripción vía RSS en el que es posible recibir alertas.

\section{Snopes}

https://www.snopes.com

Como los otros dos medios independientes estudiados, funciona como un cibermedio en el que todos los contenidos son propios. Organiza las piezas de verificación de forma similar a Chequeado o Pagella politica, con la novedad de un apartado de noticias sobre fact-checking donde se recogen las iniciativas dirigidas a fortalecer la circulación de noticias falsas. También novedoso resulta el ranking $\mathrm{Hot} 50$ en el que se clasifican los rumores que generan mayor tráfico de los lectores. Este espacio sirve para comprobar cómo algunos de estos falsos siguen gozando de gran popularidad incluso meses después de ser desmentidos. Snopes utiliza Twitter y Facebook como vías para la difusión de sus contenidos.

\section{Fact checker}

https://www.washingtonpost.com/news/fact-checker

Constituye una excepción con respecto a lo descrito hasta el momento, pues está concebida como una sección elaborada a modo de blog. Su particularidad reside en que se concede un notable protagonismo a la figura del fact-checker, encarnado en el periodista Glenn Kessler, a pesar de que son varios los profesionales que contribuyen a enriquecer la sección con sus verificaciones. Mediante textos breves de unas 500 palabras, con un estilo más informal, se analizan las declaraciones de los principales protagonistas de la esfera política estadounidense y se complementan con vídeos y enlaces a fuentes oficiales. Aunque el blog es la principal plataforma, los fact-checks se difunden también en la página Fact checker de Facebook y en la cuenta personal de Kessler en Twitter.

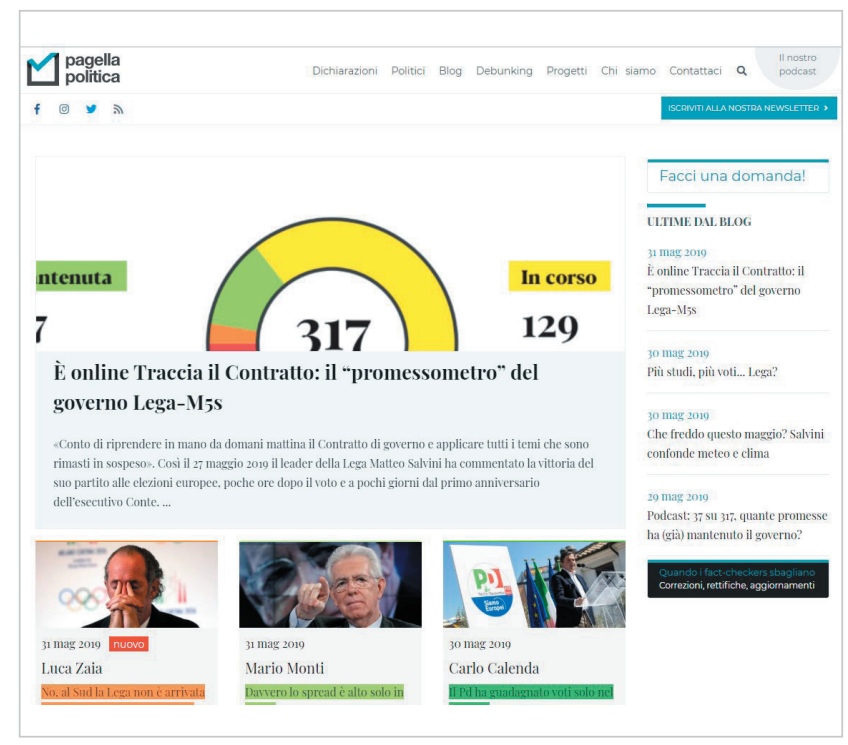

https://pagellapolitica.it

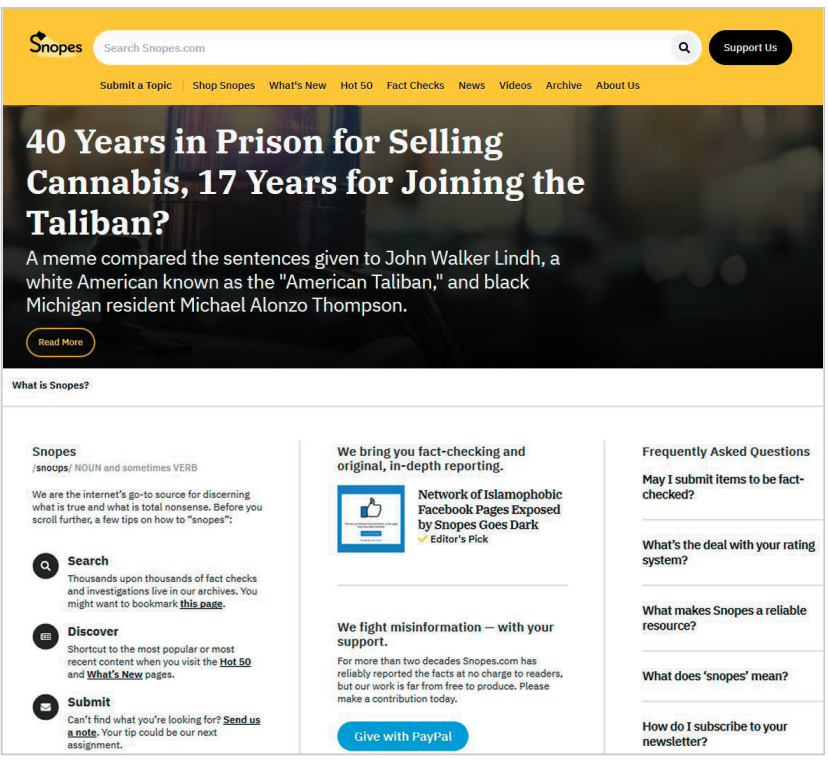

https://pagellapolitica.it

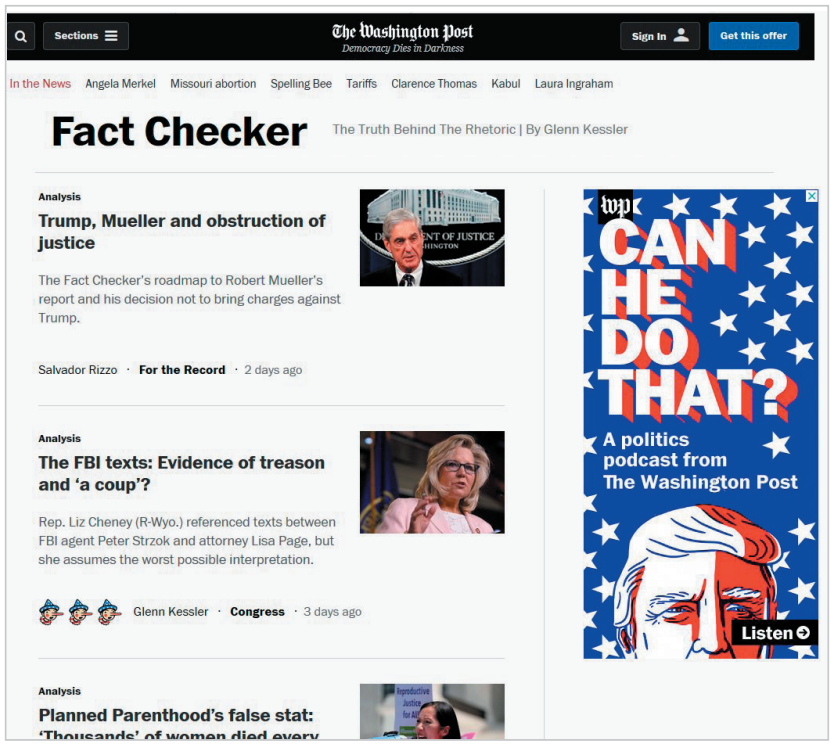

https://www.washingtonpost.com/news/fact-checker 


\section{Método de verificación}

Tabla 1. Métodos de verificación en los casos seleccionados

\begin{tabular}{|c|c|c|c|c|}
\hline \multirow{3}{*}{ Nombre } & \multicolumn{4}{|c|}{ Método de verificación } \\
\hline & \multirow{2}{*}{$\begin{array}{l}\text { Explicación } \\
\text { textual }\end{array}$} & \multicolumn{3}{|c|}{ Escala de verificación } \\
\hline & & Textual & Visual & Cromática \\
\hline $\mathrm{BBC}$ reality check & $\checkmark$ & & & \\
\hline Chequeado & $\checkmark$ & $\checkmark$ & & \\
\hline Les décodeurs & $\checkmark$ & & & \\
\hline Pagella politica & $\checkmark$ & $\checkmark$ & & $\checkmark$ \\
\hline Snopes & $\checkmark$ & $\checkmark$ & $\checkmark$ & $\checkmark$ \\
\hline Fact checker & $\checkmark$ & $\checkmark$ & $\checkmark$ & \\
\hline
\end{tabular}

Los medios seleccionados emplean diferentes fórmulas para dar cuenta del grado de precisión de las informaciones verificadas. $B B C$ reality check y Les décodeurs emplean el texto como elemento principal para calificar una información como verdadera o falsa, explicando cuáles son los datos incorrectos y cuáles los verídicos, tal y como se recoge en la tabla 1. Sin embargo, además de la mera explicación textual de los errores contenidos en una declaración, los medios especializados en fact-checking han desarrollado

Las escalas de verificación combinan términos, colores y símbolos para una lectura ágil

una serie de escalas de verificación basadas en el uso independiente o combinado de términos, colores y símbolos. Así, Chequeado opta por el empleo de una escala de verificación textual en la que los contenidos pueden ser clasificados en una escala de "verdadero" a "falso", incluyendo diferentes matices intermedios como "verdadero pero..." o "exagerado".

Pagella politica y Fact checker combinan dos elementos en sus escalas de verificación. La iniciativa italiana suma a su escala textual de cinco peldaños una cromática que comienza con dos tonos de verde seguidos por el amarillo, el naranja y el rojo para los contenidos etiquetados como "panzana pazzesca" (mentira loca, disparate). Fact checker cuenta también con una escala textual que incluye de uno a cuatro Pinocchios según el grado de falsedad del contenido, The Geppeto checkmarck para las declaraciones verídicas, el Upside-down Pinocchio para aquellas declaraciones que constituyen un cambio de idea del mismo protagonista y el Veredict pending otorgado a aquellos contenidos que resulta imposible verificar. En diciembre de 2018 se añadió una nueva categorización, el Bottomless Pinocchio, concedido a aquellas citas falsas repetidas más de veinte veces. Fact checker complementa cada una de estas categorías con símbolos que las definen, tal como puede observarse en la figura 7.

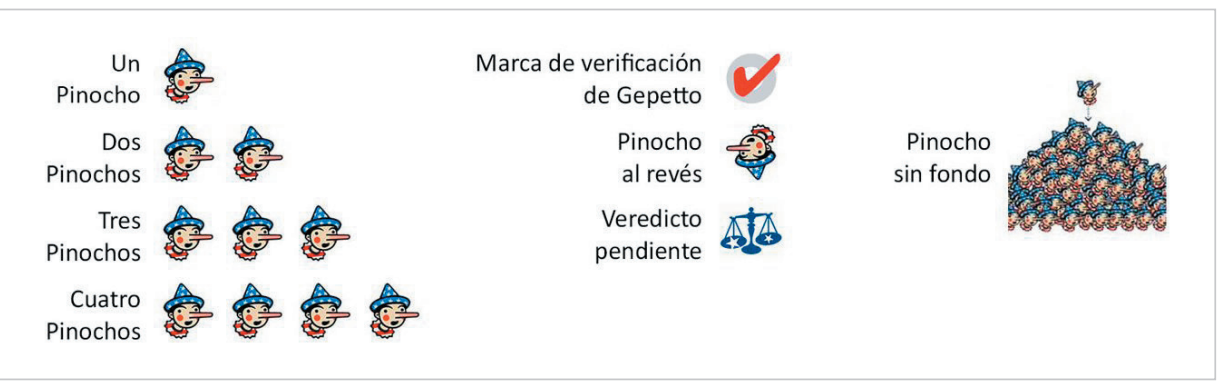

Finalmente, Snopes es el medio que dota sus escalas de verificación de una mayor riqueza a través del empleo de doce categorías con sus diferentes símbolos (figura 8). Además, cada uno de los símbolos empleados aparece destacado en color verde - para aquellos conteni-

Figura 7. Escala empleada por Fact checker

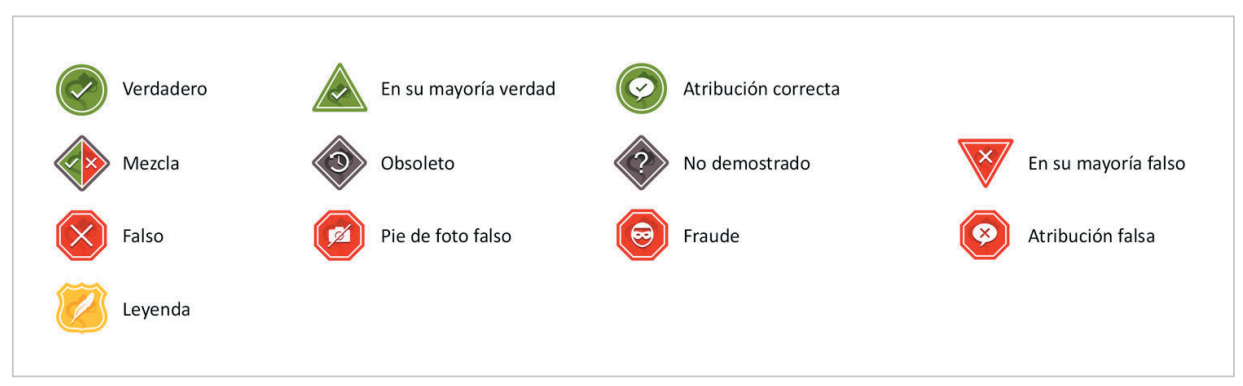

Figura 8. Escala empleada por Snopes dos verdaderos-, rojo - para los falsos-, amarillo - para los clasificados como leyendas - o gris en el caso de las informaciones no probadas o fuera de fecha. 


\section{Conclusiones}

La incorporación de las tecnologías actuales a los procesos de verificación de la información ha supuesto un paso adelante en la mejora de la calidad de la información y del debate público. Las estrategias de fact-checking, con sus fortalezas y debilidades evidenciadas en las iniciativas analizadas, han mostrado su utilidad en el campo periodístico. Del análisis realizado se concluye que, a pesar del creciente interés que la práctica del fact-checking ha despertado durante los últimos años, muchos de los proyectos no han gozado de la continuidad necesaria. Gracias a esta investigación ha sido posible identificar que un tercio de las iniciativas revisadas no mostraron actividad en los tres meses previos a su análisis.

Atendiendo a la distribución geográfica del fact-checking, ésta parece ser una práctica anclada en el periodismo europeo y norteamericano. Europa, Estados Unidos y Canadá acogen 86 de los 135 fact-checkers con actividad en el período de análisis. Éstos, además, han hecho de la Web el principal canal para dar cuenta de sus verificaciones, pues el $70 \%$ de los proyectos son medios nativos digitales.

El período comprendido entre 2012 y 2017 constituye la etapa de mayor auge de iniciativas de fact-checking. Durante estos años aparecieron más de 100 espacios de verificación cuyo foco se centra fundamentalmente en la información nacional. Lo hacen con una estructuración clásica de los contenidos, al estilo de una noticia que resuelve un interrogante o precisa un dato o afirmación. Integran fuentes originales y destacan por la riqueza y diversidad en el uso de mecanismos de verificación que combinan elementos de naturaleza diversa - textual, icónica, cromática-. Algunos de estos sistemas se diseñan ex profeso para responder a los objetivos del medio. Sin embargo se concluye que, por el momento, el texto es el elemento principal en la verificación, ya que el uso de otro tipo de elementos está todavía en fase de experimentación para la mayoría de los verificadores.

Este estudio ha servido, además, para identificar los patrones empleados por seis fact-checkers de referencia en contextos idiomáticos, económicos y culturales diferentes. Si bien los casos seleccionados no representan la realidad global del fact-checking - lo cual constituye una limitación para el estudio presentado-, son útiles para observar las fórmulas que pueden ser empleadas para alcanzar el objetivo de la verificación de la información en el contexto actual. Una vía, no la única, donde se aplica la innovación para hacer frente al fenómeno de las fake news, de especial impacto en la sociedad red.

\section{Notas}

1. La base de datos del Duke Reporters' Lab está coordinada por los profesores Mark Stencel y Bill Adair. Sus criterios fundamentales son que las plataformas cuenten con un propósito informativo y desarrollen su actividad con transparencia de fuentes, métodos y financiación. En su sitio web explican cómo identifican los fact-checkers: https://reporterslab.org/how-we-identify-fact-checkers

2. El código de principios de la Red Internacional de Verificación de la Información (IFCN, en inglés) hace referencia a la independencia, la política abierta de correcciones y la transparencia de fuentes, financiación y método. Los criterios pueden ser consultados en:

https://ifcncodeofprinciples.poynter.org/know-more/the-commitments-of-the-code-of-principles

3. La relación de fact-chekers analizados se encuentra disponible en el repositorio de acceso abierto Figshare: https://doi.org/10.6084/m9.figshare.7854938.v1

\section{Referencias}

Bauman, Zygmunt (2007). Liquid modernity. Cambridge: Polity Press. ISBN: 9780745624105

Beckett, Charlie; Deuze, Mark (2016). "On the role of emotion in the future of journalism”. Social media + society, v. 2, n. 3.

https://doi.org/10.1177/2056305116662395

Berinsky, Adam J. (2015). "Rumors and health care reform: Experiments in political misinformation". British journal of political science, v. 47, n. 2, pp. 241-262.

https://doi.org/10.1017/S0007123415000186

Bloch, Marc (1999). Historia e historiadores. Madrid: Akal. ISBN: 9788446010371

Brandtzaeg, Petter-Bae; Følstad, Asbjørn; Chaparro-Domínguez, María-Ángeles (2018). “How journalists and social media users perceive online fact-checking and verification services". Journalism practice, v. 12, n. 9, pp. $1109-1129$. https://doi.org/10.1080/17512786.2017.1363657

Bryman, Alan (2006). “Integrating quantitative and qualitative research: How is it done?”. Qualitative research, v. 6, n. 1, pp. 97-113.

https://doi.org/10.1177/1468794106058877 
Carpentier, Nico (2011). Media and participation. A site of ideological-democratic struggle. Bristol: Intellect. ISBN: 978 1841504070

https://oapen.org/download?type=document\&docid $=606390$

Casero-Ripollés, Andreu (2012). "Beyond newspapers: News consumption among young people in the digital era". Comunicar, v. 20, n. 39, pp. 151-158.

https://doi.org/10.3916/C39-2012-03-05

Castells, Manuel (2001). The internet galaxy. Oxford: Oxford University Press. ISBN: 9780199255771

Chadwick, Andrew (2013). The hybrid media system: Politics and power. Oxford: Oxford University Press. ISBN: 9780 199759484

Cherubini, Federica; Graves, Lucas (2016). The rise of fact-checking sites in Europe. Reuters Institute for the Study of Journalism, University of Oxford.

http://reutersinstitute.politics.ox.ac.uk/our-research/rise-fact-checking-sites-europe

Currie-Sivek, Susan; Bloyd-Peshkin, Sharon (2018). "Where do facts matter?". Journalism practice, v. 12, n. 4, pp. $400-421$. https://doi.org/10.1080/17512786.2017.1307694

Deuze, Mark (2004). “What is multimedia journalism?”. Journalism studies, v. 5, n. 2, pp. 139-152.

https://doi.org/10.1080/1461670042000211131

Duke Reporters' Lab (2018). Fact-checking.

https://reporterslab.org/fact-checking

Elizabeth, Jane (2014). Who are you calling a fact checker?. American Press Institute.

https://www.americanpressinstitute.org/fact-checking-project/fact-checker-definition

Fidler, Roger (1997). Mediamorphosis. Understanding new media. Thousand Oaks, CA: Pine Forge Press. ISBN: 9780 803990869

Franklin, Bob (2011). The future of journalism. London: Routledge. ISBN: 9780415629294

Gaitán-Moya, Juan-Antonio; Piñuel-Raigada, José-Luis (1998). Técnicas de investigación en comunicación social. Madrid: Síntesis. ISBN: 9788499583570

George-Palilonis, Jennifer (2012). The multimedia journalism. Storytelling for today's media landscape. Oxford: Oxford University Press. ISBN: 9780199764525

Glück, Antje (2016). "What makes a good journalist? Empathy as a central resource in journalistic work practice”. Journalism studies, v. 17, n. 7, pp. 893-903.

https://doi.org/10.1080/1461670X.2016.1175315

Graves, Lucas (2016). Deciding what's true: The rise of political fact-checking in American journalism. New York: Columbia University Press. ISBN: 9780231175074

Gueham, Farid (2017). Le fact-checking: une réponse à la crise de l'information et de la démocratie. Paris: Fondation pour l'innovation politique.

http://www.fondapol.org/etude/farid-gueham-le-fact-checking-une-reponse-a-la-crise-de-linformation-et-de-la-democratie

Hermida, Alfred; Thurman, Neil (2008). "A clash of cultures. The integration of user-generated content within professional journalistic frameworks at British newspaper websites". Journalism practice, v. 2, n. 3, pp. 343-356.

https://doi.org/10.1080/17512780802054538

Holton, Avery E.; Lewis, Seth C.; Coddington, Mark (2016). "Interacting with audiences. Journalistic role conceptions, reciprocity, and perceptions about participation". Journalism studies, v. 17, n. 7, pp. 849-859.

https://doi.org/10.1080/1461670X.2016.1165139

Hujanen, Jaana (2016). "Participation and the blurring values of journalism". Journalism studies, v. 17, n. 7, pp. 871-880. https://doi.org/10.1080/1461670X.2016.1171164

Jones-Patterson, Maggie; Urbanski, Steve (2006). "What Jayson Blair and Janet Cooke say about the press and the erosion of public trust". Journalism studies, v. 7, n. 6, pp. 828-850.

https://doi.org/10.1080/14616700600980488

Kovach, Bill; Rosenstiel, Tom (2001). The elements of journalism: What news people should know and the public should expect. New York: Three Rivers Press. ISBN: 9780804136785

Lopez-Borrull, Alexandre; Vives-Gràcia, Josep; Badell, Joan-Isidre (2018). "Fake news, ċamenaza u oportunidad para los profesionales de la información y la documentación?". El profesional de la información, v. 27, n. 6, p. 1346-1356.

https://doi.org/10.3145/epi.2018.nov.17 
Mantzarlis, Alexios (2018). "Fact-checking 101". In: Ireton, Cherilyn; Posetti, Julie. Journalism, fake news \& disinformation: Handbook for journalism education and training. Francia: Unesco, pp. 85-100. ISBN: 9789231002816 https://unesdoc.unesco.org/ark:/48223/pf0000265552

McBride, Kelly; Rosenstiel, Tom (2013). The new ethics of journalism: Principles for the $21^{\text {st }}$ Century. Thousand Oaks: CQ Press. ISBN: 9781604265613

Micó-Sanz, Josep-Lluís; Pérez-Soler, Susana (2015). "La participación en los medios de comunicación a través de Twitter. Estudio comparativo entre los periodistas catalanes y belgas”. Sur le journalisme, v. 4, n. 2, pp. 62-73.

https://surlejournalisme.com/rev/index.php/slj/article/view/218

Paulussen, Steve; Heinonen, Ari; Domingo, David; Quandt, Thorsten (2007). “Doing it together: Citizen participation in the professional news making process". Observatorio (OBS*), v. 1, n. 3.

http://obs.obercom.pt/index.php/obs/article/view/148

Pavlik, John (2000). "The impact of technology on journalism". Journalism studies, v. 1, n. 2, pp. 229-237.

https://doi.org/10.1080/14616700050028226

Poynter (2018). International fact-checking network. Code of principles.

https://ifcncodeofprinciples.poynter.org

Roth, Yoel (2018). "Automation and the use of multiple accounts". Blog.twitter.com, 21 Febr.

https://blog.twitter.com/developer/en_us/topics/tips/2018/automation-and-the-use-of-multiple-accounts.html

Salaverría, Ramón (2005). Redacción periodística en internet. Pamplona: Eunsa. ISBN: 8431322594

http://dadun.unav.edu/handle/10171/34331

Salaverría, Ramón (2015). "Ideas para renovar la investigación sobre medios digitales". El profesional de la información, v. 24, n. 3, p. 223.

https://doi.org/10.3145/epi.2015.may.01

Singer, Jane B. (2005). "The political j-blogger: Normalizing a new media form to fit old norms and practices". Journalism: theory, practice \& criticism, v. 6, n. 2, pp. 173-198.

https://doi.org/10.1177/1464884905051009

Van-der-Haak, Bregtje; Parks, Michael; Castells, Manuel (2012). "The future of journalism: Networked journalism". International journal of communication, v. 6, p. 16.

https://ijoc.org/index.php/ijoc/article/view/1750/832

Wardle, Claire; Derakhshan, Hossein (2017). Information disorder: Toward an interdisciplinary framework for research and policy making. Strasburg: European Council.

https://edoc.coe.int/en/media/7495-information-disorder-toward-an-interdisciplinary-framework-for-research-andpolicy-making.html

Zubiaga, Arkaitz; Liakata, Maria; Procter, Rob; Hoi, Geraldine-Wong-Sak; Tolmie, Peter (2016). “Analysing how people orient to and spread rumours in social media by looking at conversational threads". PLoS one, v. 11, n. 3, p. e0150989. https://doi.org/10.1371/journal.pone.0150989

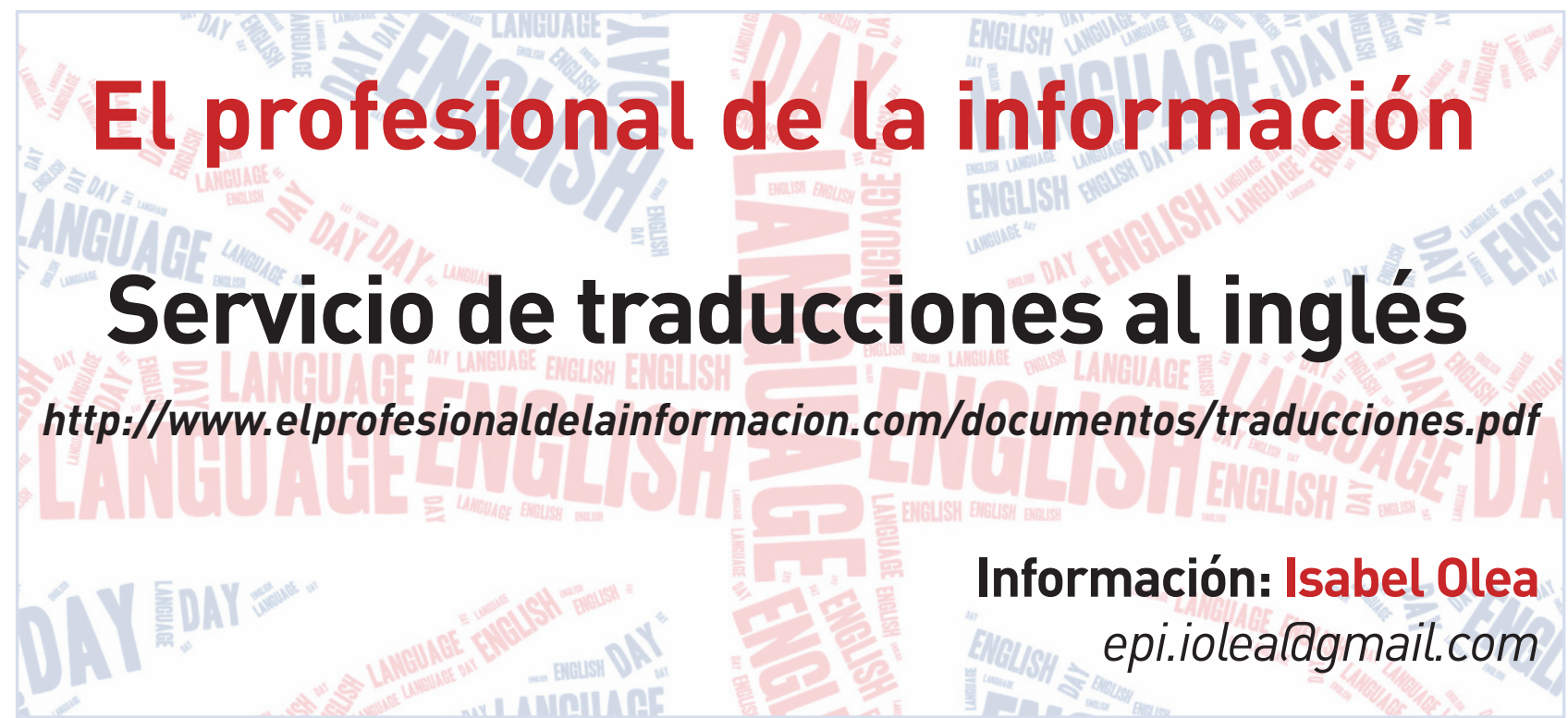

